\title{
Formation of Arching Flux Tubes at the Base of the Solar Convection Zone
}

\author{
Y. Fan \\ High Altitude Obs., National Center for Atmospheric Research, 3450 \\ Mitcheil Lane, Boulder, CO 80301, USA
}

\begin{abstract}
Solar active regions are believed to correspond to the topmost portions of $\Omega$-shaped arching flux tubes that have risen buoyantly from the base of the solar convection zone, where strong toroidal magnetic fields are being generated by the dynamo process. The development of such emerging $\Omega$-loops is likely a result of the buoyant instability associated with the submerged toroidal magnetic field. Using an anelastic MHD code, we simulate the formation of buoyant, arching flux tube structures as a result of the non-linear growth of the undular instability of a neutrally buoyant layer of horizontal, unidirectional magnetic field at the base of the solar convection zone.
\end{abstract}

\section{Introduction}

It has been shown (see e.g. Newcomb 1961; Hughes \& Cattaneo 1987) that a horizontal magnetic field, embedded in a gravitationally stratified fluid may be subject to both the two-dimensional interchange instability (without bending the field lines), as well as the three-dimensional undular instability (with nonzero wave numbers in both horizontal directions perpendicular and parallel to the magnetic field). For the 3D undular modes, motion along the field lines is allowed and it can be diverging at the crests and converging at the troughs, unburdening the raised portions and increasing the burden at the troughs, thereby amplifying the undulation.

The magnetic buoyancy instabilities of a horizontal magnetic layer has been investigated in many previous numerical simulations, both in 2D (e.g. Cattaneo \& Hughes 1988; Cattaneo, Chiueh, \& Hughes 1988) and in 3D (e.g. Matthews, Hughes \& Proctor 1990). In these calculations, the initial equilibrium magnetic layer is most unstable to the 2D interchange modes. We on the other hand consider an equilibrium magnetic layer that is in the state of neutral buoyancy such that it is only unstable to $3 \mathrm{D}$ undular modes but stable to $2 \mathrm{D}$ pure interchange modes. In this way, we study the formation of arching tubes by the growth of the 3D undular modes without the complication of the presence of the 2D instabilities. Recent study of the storage of toroidal magnetic field (Rempel, Schüssler, \& Tóth 2000) shows that an extended layer of strong toroidal magnetic field located in a weakly subadiabatic overshoot layer attains an equilibrium that is very close to the state of neutral buoyancy. 

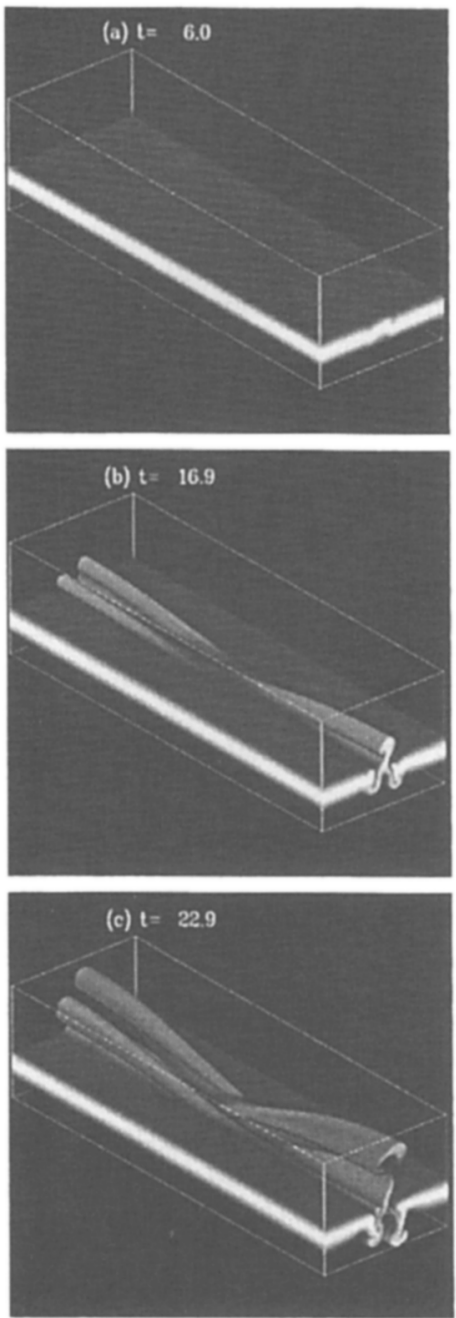
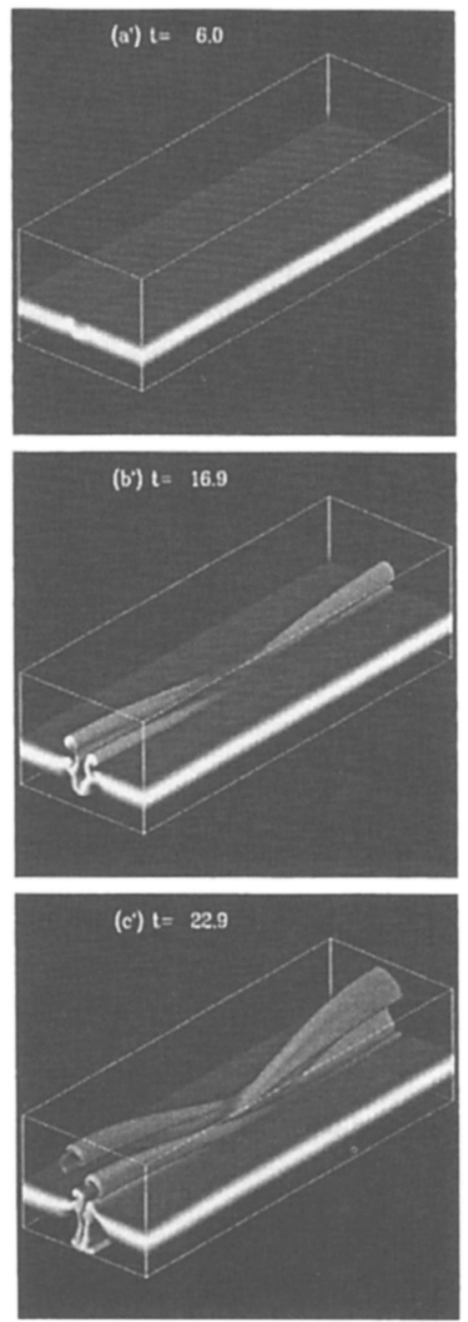

Figure 1. Formation of buoyant, arching flux tubes from the equilibrium magnetic layer perturbed with a localized velocity field. The case shown here is the same as that shown in Figure 13 of Fan (2000), except that the perturbing velocity field here is asymmetric. The longer horizontal dimension of the box is along the $x$-direction, which is the direction of the initial magnetic field. The left and right columns show respectively the evolution as viewed from two different perspectives. 


\section{Results}

Our simulations start with a layer of horizontal, unidirectional magnetic field embedded in an adiabatically stratified polytrope, with a density stratification that remains unchanged from that of the background polytrope (i.e. in a neutral buoyant state). Gas pressure and temperature are reduced in the magnetic layer to satisfy the condition of hydrostatic equilibrium. The linear stability analysis of the initial equilibrium and the detailed results of our numerical simulations can be found in Fan (2000). Figure 1 illustrates an example simulation of the formation of buoyant arching flux tubes from the equilibrium magnetic layer.

The results of our simulations are summarized as follows. We find that distinct arching flux tubes form as a result of the growth of the 3-D undular instability. The apexes of the arching tubes become increasingly buoyant due to the diverging mass flow from the apexes to the troughs. There is significant stretching of the field lines at the loop apex and as a result the decrease of field strength with height is found to follow approximately $B \propto \rho^{0.5}$, instead of the $B \propto \rho$ relation which is expected for the rise of a straight horizontal tube. This variation of $B$ with height is in agreement with the result of previous thin flux tube simulations of the Parker instability of emerging $\Omega$-loops during the rise across the lower half of the solar convection zone (e.g. Moreno-Insertis 1992). Even though the initial horizontal magnetic field is untwisted, the upward moving cross-sections of the arching tubes maintain their cohesion as they rise through the distance of about one density scale height included in the simulation domain. The magnetic tension resulting from bending the field lines of the $\Omega$ loop can effectively oppose the vorticity generation by buoyancy in most of the interior of the tube cross-section, and prevent the formation of two dominant vortex rolls as seen in previous 2-D simulations of the rise of untwisted horizontal tubes. In addition, the fact that both the buoyancy and the tension forces grow self-consistently from zero as the tubes arch is also a crucial factor for the improved cohesion of the rising tubes.

\section{References}

Cattaneo, F., and Hughes, D. W. 1988, J. Fluid Mech., 196, 323

Cattaneo, F., Chiueh, T., and Hughes, D. W. 1990, J. Fluid Mech., 219, 1

Fan, Y. 2000, ApJ, in press

Hughes, D. W., and Cattaneo, F. 1987, Geophys. Astrophys. Fluid Dynamics, 39,65

Matthews, P. C., Hughes, D. W., and Proctor, M. R. E. 1995, ApJ, 448, 938

Moreno-Insertis, F., 1992, in "Sunspots: Theory and Observations", (Kluwer Academic Publisher), ed.: J. H. Thomas, and N. O. Weiss, 385

Newcomb, W. A. 1961, Phys. Fluids, 4, 391

Rempel, M., Schüssler, M., and Tóth, G. 2000: A\&A, in press 